\title{
Chapter 6 \\ Integrating Extended Non-destructive Testing in the Life Cycle Management of Bonded Products-Some Perspectives
}

\author{
Welchy Leite Cavalcanti, Elli Moutsompegka, Konstantinos Tserpes, \\ Paweł H. Malinowski, Wiesław M. Ostachowicz, Romain Ecault, \\ Neele Grundmann, Christian Tornow, Michael Noeske, Peter Schiffels, \\ and Bernd Mayer
}

\begin{abstract}
In this chapter, we outline some perspectives on embracing the datasets gathered using Extended Non-destructive Testing (ENDT) during manufacturing or repair process steps within the life cycle of bonded products. Ensuring that the ENDT data and metadata are FAIR, i.e. findable, accessible, interoperable and re-usable, will support the relevant stakeholders in exploiting the contained material-related information far beyond a stop/go decision, while a shorter time-to-information will facilitate a prompter time-to-decision in process and product management. Exploiting the value of ENDT (meta)data will contribute to increased performance by integrating all defined, measured, analyzed and controlled aspects of material transformation across process and company boundaries. This will facilitate the optimization of manufacturing and repair operations, boosting their energy efficiency and productivity. In this regard, some aspects that are currently driving activities in the field of pre-process, in-process and post-process quality assessment will be addressed in the following. Furthermore, some requirements will be contemplated for harmonized and conjoint data transfer ranging from a bonded product's beginning-of-life through its end-oflife, the customization of stand-alone or linked ENDT tools, and the implementation of sensor arrays and networks in joints, devices and structural parts to gather material-related data during a product's middle-of-life application phase, thereby fostering structural health monitoring (SHM).
\end{abstract}

W. Leite Cavalcanti $(\varangle) \cdot$ N. Grundmann $\cdot$ C. Tornow $\cdot$ M. Noeske $\cdot$ P. Schiffels $\cdot$ B. Mayer Fraunhofer Institute for Manufacturing Technology and Advanced Materials IFAM, Wiener Str. 12, 28359 Bremen, Germany

e-mail: welchy.leite.cavalcanti@ifam.fraunhofer.de

E. Moutsompegka $\cdot$ K. Tserpes

Laboratory of Technology \& Strength of Materials, Department of Mechanical Engineering \& Aeronautics, University of Patras, 26500 Patras, Greece

P. H. Malinowski · W. M. Ostachowicz

Institute of Fluid-Flow Machinery, Polish Academy of Sciences, Fiszera 14, Gdańsk 80231,

Poland

R. Ecault

Airbus Operations S.A.S., 316, Route de Bayonne, B.P. D4101, 31060 Cedex 9 Toulouse, France

(C) The Author(s) 2021

W. Leite Cavalcanti et al. (eds.), Adhesive Bonding of Aircraft Composite Structures,

https://doi.org/10.1007/978-3-319-92810-4_6 
Keywords Life cycle management $\cdot$ FAIR ENDT data and metadata $\cdot$ Harmonized characterization data (CHADA) documentation - Customisation · Standardization • Sensor network

\subsection{Introduction}

One of the foremost ongoing trends and initiatives is not only that data are collected and exchanged for documentation purposes along a process or value chain, but also that value can be added if the substantial information contained in these data is extracted by the providers and owners. This typically means evaluating several sets of interconnected metadata, measured characterization data or simulation-based modelling data that constitute knowledge sources.

In the first of the subsequent sections, we accentuate the technical benefits of consistently linking joints with their associated material-related data. We base our selection of potential prospects on our perception of the distinctive prevailing global transport of material goods, combination of logistic processes and transfer of ideas along with the life cycle of material products such as adhesive joints. From this network point of view, the urgent need for composite product data interoperability for life cycle management and the sustainment of aircraft fleets was recently highlighted by McMillan et al. [1]. Next, we highlight how the balancing of customization and efficiency will profit from a certain readiness for standardization and from establishing the consistency of (meta)data formats. This will help overcome the need for data translation at each interface [2]. In this context, we present the outline of a research project that resulted in the successful development and implementation of a customized hand-held aerosol wetting test (AWT) device relying on the core elements presented in the ComBoNDT project [3]. After that, we introduce stepwise approaches for integrating ENDT investigations using optically stimulated electron emission (OSEE) and the space-resolved findings in, e.g., material data management systems while safeguarding data integrity. We show how the concept developed in the ComBoNDT project [3] facilitates the linking of ENDT data with design-relevant features that are significant along the life cycle of adhesive joints. Finally, we complement the latter two surface quality-related approaches through the prospect of material-integrated sensor networks and fibre-based sensors, thereby facilitating the structural health monitoring (SHM) of adhesive joints.

\subsection{Data Transfer Along the Product Life Cycle}

In Chap. 1, we introduced the image of an enhanced triangular industrial automation pyramid, in which only two of the three lateral faces of the pyramid were presented. The third face, which was hidden in the sketch due to the limited angle of view rather 
than a limited awareness, represents the environmental impact during the manufacturing phase. Therefore, we highlight the environmental aspects during the complete product life cycle in Fig. 6.1, showing the top view of a tetrahedron and sketching the incorporation of the manufacturing process as a part of a product's beginning-of-life (BoL) period. In this way, the relevance of the manufacturing characteristics for the sustainability of a product is illustrated.

In the previous chapters of this book, we demonstrated that using advanced ENDT tools and procedures facilitates the gathering of high-quality data from production or repair processes based on adhesive bonding technology to obtain high-quality products. From an overall material-related and process-comprehensive perspective, the outcomes of the United Nations Conference on Environment and Development (UNCED) in Rio de Janeiro, 1992, stressed that sustainability is the principal worldwide political goal for the future development of humankind, and thus, shall be respected during any product life cycle. This challenge encompasses environmental, economic and social (or societal) aspects. Related tools supporting researchers and developers in tackling these challenges are the internationally standardized life cycle assessment (LCA) for the environmental part, life cycle costing (LCC) for the economic assessment, which also accounts for the use and end-of-life phases, and social life cycle assessment (SLCA), which may be applied after identifying the system boundaries [4]. Product sustainability may also be captured following the holistic concept of life cycle management (LCM). As pointed out in the framework of the United Nations Environment Programme [5], "understanding, quantifying and communicating the environmental impacts and resource consumption of products" are essential steps on the way towards a "resource-efficient twenty-first century

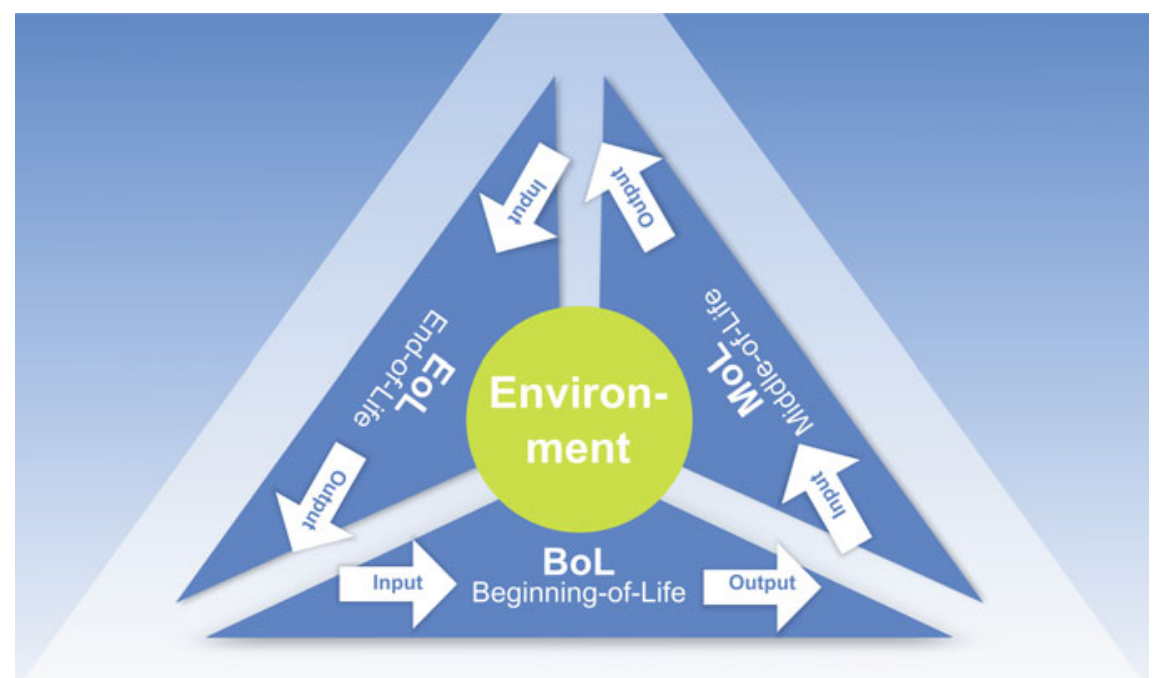

Fig. 6.1 Triangular pyramid highlighting the relevance of all product life cycle phases on a product's environmental impact 
green economy". Aggregation of the involved data at the product system level and their management in databases support an intelligent understanding and a trajectory towards sustainable development. Therefore, it is not surprising that LCA and LCC are used in the early phases of aircraft structural design based on CFRP parts [6]. Calado et al. demonstrated in the framework of a combined LCA and LCC approach that the quality control (QC) process step following the demoulding and finishing of a CFRP part may be evaluated based on the QC cycle time and the reject rate of the quality assurance step [6]. This approach throws light on the relevance of reliable and accurate ENDT procedures not only for the quality assessment of CFRP parts before the subsequent process step, but also for the ecological and economic impacts of quality assurance when designing an adhesive bonding process. Considering merely the surface quality of a CFRP adherend, space-resolving ENDT tools facilitating a local cleaning on demand, while also largely avoiding false negatives within the quality assessment, will guide and support process engineers in choosing an appropriate and sustainable pre-treatment in the case of a reject during QC. Beyond that, we infer that the life cycle-based sustainability assessment of adhesively bonded products will largely profit from ENDT procedures and interoperable ENDT datasets, for instance, for optimizing future material-related process steps or due to the availability of joint-specific data that can guide individualized decisions during maintenance, repair and overhaul (MRO) and the end-of-life phase.

Avoiding waste during all the life cycle phases of a product will contribute to greatly enhancing its performance from a twenty-first century perspective. As Otte et al. point out with respect to manufacturing, the "ability to quickly access and share data is key to optimizing and streamlining any industrial production process" [7]. Following the apparently linear time axis in Fig. 1.4 (Chap. 1), which points from input towards output in the material transformation process during adhesive bonding, we would like to go beyond the view of a local manufacturing or repair process and outline some examples of worldwide data-based workflows based on interlinking several such pyramids and exchanging data from various sources and with distinct data quality. For such global scenarios, we would like to draw upon aspects of the nine Industry 4.0 clusters identified by Hermann et al. [8], namely interconnection, collaboration, standards, security, data analytics, information provision, decentralized decisions, physical assistance and virtual assistance. Referenced by worldwide and European initiatives, holistic life cycle-based thinking and acting are gaining meaning and visibility, thus embracing the putative perception of a finally ending sequence of subsequent phases in a product's life through a life cycle within a more holistic conception, as depicted in Fig. 6.1. Therefore, in the more general perception, such circular reasoning is, on the one hand, throwing light on connecting the end-oflife (EoL) and beginning-of-life (BoL) scenarios of products from the material-based technical, social, ecological and economic points of view comprising sustainable development. On the other hand, maintenance and repair, as essential user cases during the middle-of-life (MoL) life cycle phase [7], are gaining attention in light of product life cycle assessment (LCA) procedures. Pragmatically, this means that for any form of EoL recycling, material-related information on the BoL or MoL actions and their effects shall be seminal. Material-related data that meet FAIR data principles 
and that are straightforwardly gathered during manufacturing or repair processes are pathbreaking in this sense. The interoperability of exchanged data facilitates knowledge management based on necessary ontologies like Basic Formal Ontology (BFO) [7] or the recently developed European Materials Modelling Ontology (EMMO) for applied sciences [9, 10]. EMMO is integrated into virtual marketplaces like VIMMP [11, 12] or MarketPlace [13] and allows end-users to represent their manufacturing process and innovation challenges in a standard ontological form. This is supported by smart targeted guidance through the whole translation process to "deliver a full complete experience to companies", profiting from an ontology-based open translation environment grounded in EMMO [2].

From the material point of view, we would like to accentuate that in adhesive bonding technology, the quality and durability of joints are crucially determined by the scale-comprehensive effects of molecular interactions and mesoscopic transport processes during the formation of adhesion between adherends and the adhesive system [14]. On the one hand, we would like to express our expectation that ontologies will be the foundation for combining physics-based and data-driven approaches in order to assess more deeply the formation of adhesion and its time-dependent development during the BoL and MoL phases of bonded joints and the respective products, thus contributing to more sustainable material development. On the other hand, we would also like to exemplify that evaluating the ENDT datasets of prebond adherend surfaces and adhesively bonded joints continues to advance and is increasingly assisting in cleaning and joining processes in a growing number of user cases.

From the process point of view, we estimate that the abundance of adherend materials and their geometrical shapes, surface pre-treatment process steps, adhesive systems (multi-layered and multi-component), application tools and implementations of the bonded joints involving distinct environments in production, maintenance and repair processes will drive the need for technological adaptation and the demand for information exchange. We expect further customization of ENDT tools and an increasingly individual definition and realization of their interfaces with the surrounding network of catenated integrants of quality assurance systems, e.g. with further NDT tools or by having control personnel organized within the automation pyramid. From our point of view, a systematic common language based on shared top-level or domain-specific ontologies will greatly support the directed and unambiguous communication and exchange of findable, accessible, interoperable and reusable data and information. 


\subsection{Customization and Further Advancement of ENDT Tools and Procedures}

Based on the available knowledge, e.g. that achieved within the ENCOMB and ComBoNDT research projects, and driven by market reviews and customer feedback for detailing the technical and economic user case aspects, interested stakeholders may instigate further developments along with the initiated ideas, thereby contributing to innovative ENDT procedures in the third decade of the twenty-first century. Here, we would like to show through examples that the customization of ENDT tools and procedures is, on the one hand, based on the elaborated, documented and available outcomes of the last decade, at the end of which we performed the developments described in the following. On the other hand, such customization further facilitates tailored and improved customer interaction and increased customer satisfaction.

In the previous chapters, we presented how customizing the operands was achieved in the ComBoNDT research project by configuring a set of CFRP adherends with different surface states. In addition, both the ENDT tools and the respective procedures were technologically advanced. Beyond that, their customization while following standardized approaches is a frequent challenge for studies focusing on operator-related aspects. We would like to highlight here that within the consortium of the ComBoNDT joint research project, this line of action was followed not only by the contributing research institutions, but especially by the small and medium-sized enterprise (SME) partners involved. Significantly, a fast and targeted response to the increasing market demand for NDT technologies is being provided by innovative SMEs [15].

In the following, we highlight the findings of the recently completed HANOB joint research project, involving partners from institutional and SME research and development (R\&D), which developed a portable ENDT device used to perform a handheld wetting test $[16,17]$. Hereby, a portable measuring system to monitor the wettability of surfaces was designed and developed, and a prototype setup was constructed. The basic components comprise an aerosol nozzle for generating a welldefined spray pattern of pure water, LED-based illumination, and a compact camera for capturing the approx. $10 \mathrm{~cm}$ wide droplet pattern resulting in the substrate being inspected, as sketched in Fig. 6.2.

With this easy-to-use setup, it is possible to detect, analyze and objectively evaluate changes in the wettability of components. For technologically relevant user cases, it was also demonstrated that the effectiveness of the performed surface pre-treatment procedures can be mapped and the presence of contaminants can be detected. The developed compact prototype enabled a reliable surface quality inspection to be performed on intricately accessible surface regions and locally pre-treated substrate surfaces. The manipulation and operation of the device, as well as user prompting through a newly developed intuitive graphical interface operated via a touchscreen, were designed to facilitate easy operation after brief operator training. 


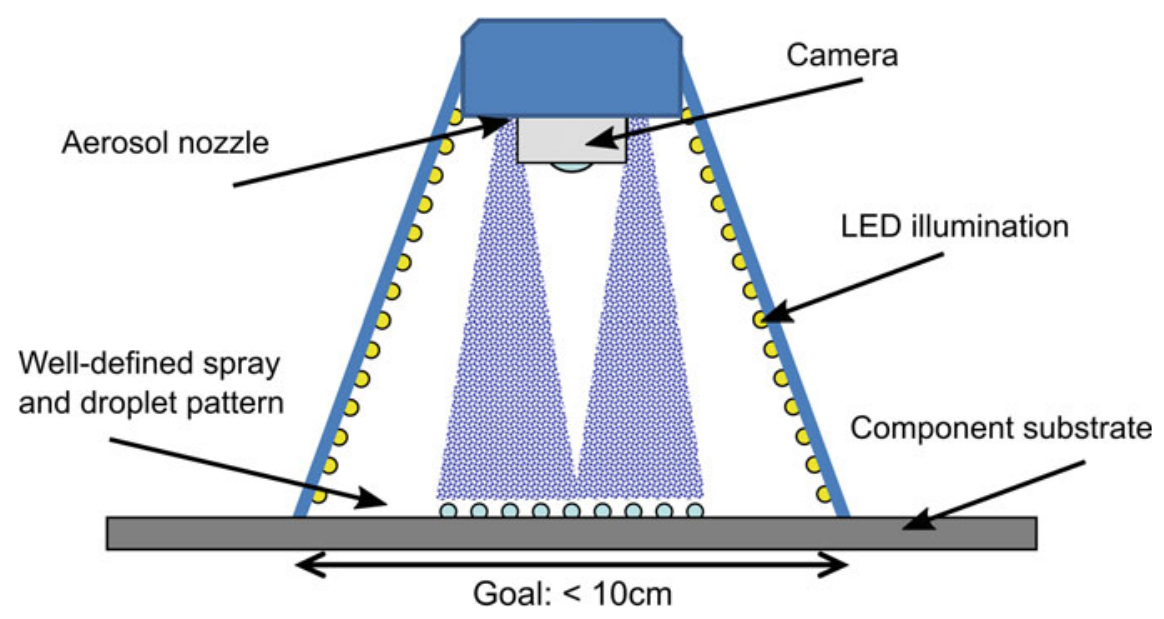

Fig. 6.2 Sketch of a hand-held customized aerosol wetting test device

Once it has been further developed to achieve the readiness level of an industrial device, this portable setup will provide an advantageous cost/performance ratio and will be deployable not only in production, e.g. for the inspection of target areas, but also in the field during repair processes in applications far exceeding aeronautical user cases. Nonetheless, the example application we highlight in Fig. 6.3 shows the HANOB demonstration setup during the inspection of a wing flap. Applied in such demanding environments, this portable device will bridge visual inspection with the naked eye and automated quality assessment.

As both the portable prototype and the industrial robot-aided AWT devices for automated large area inspection yield complex yet similar datasets, we anticipate that standardizing the data acquisition and analysis and further developing data postprocessing options for AWT findings will amplify the application portfolio of this
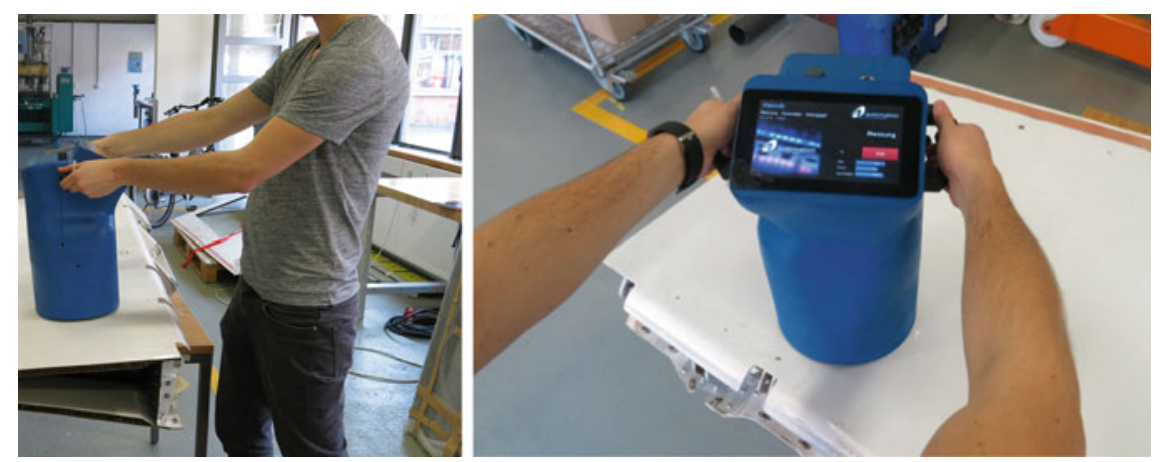

Fig. 6.3 HANOB demonstration setup operated for the inspection of a wing flap from Airbus Germany 
ENDT method. Based on the insights and experience gained in the ComBoNDT project reported in Chap. 5, we anticipate that robust physics-based or data-driven post-processing procedures will contribute to increasing the process reliability in coating, painting and bonding applications. For example, our probability of detection (POD) investigations of the AWT datasets obtained for CFRP surfaces showed that in the release agent contamination scenario, the performance of the testing procedure depended on the selection of the characteristic image feature to be evaluated. We revealed a better detection performance when using the feature of wettability instead of using the feature of droplet diameter. As a next step, from the data postprocessing point of view, we suggest performing a POD calculation by coupling the two approaches to determine whether the performance can be advanced even further. From the test setup point of view, the area density of the applied aerosol droplets might be further adapted to be optimum for this scenario. Thereby, an automatized optimization of the monitoring process, e.g. by tuning both the aerosol deposition and the feature-dependent detection performance, could facilitate reproducibly adjusted and customized inspection settings. We would like to highlight that at the end of the day, the multi-dimensional optimization of several parameters would be desirable, which would require extended modelling resources. Thereby, the advantageous interplay between quality assurance and digitalization is showcased.

\subsection{Harmonized Presentation of ENDT Data and Metadata}

Currently, the European Commission is orchestrating several ongoing European initiatives to establish an open innovation environment that, from our point of view, will also have a substantial impact on product quality control. We base these prospects on, for instance, the discernible progress in establishing a standard data structure for materials characterization (i.e. CHAracterization DAta (CHADA) [18]) and materials modelling (i.e. MOdellingDAta (MODA) [19]) with a range of potentially very different approaches and methods. We highlight here examples of those activities under the umbrella of the European Commission, which are embodied by the interacting [20] European Materials Modelling Council (EMMC) [21, 22] and the European Materials Characterisation Council [23], both of which have gained high visibility due to their agile web presence.

As an example, in Fig. 6.4, we suggest an approach for a CHADA documentation implying metadata aspects for the inspection of a CFRP part using the ENDT technique known as optically stimulated electron emission (OSEE). We would like to highlight that such an approach exceeds that described in Chap. 1 due to its more comprehensive and standardization-oriented setting. Moreover, embracing the physics of material interactions, as described by Romanos et al. [18] for impacting probes (here: ultraviolet light) and detected probes (here: photoelectrons), may be perceived as an expression of a physics-based consideration of the material properties and the characterization process itself. We may consider a physics-based approach 


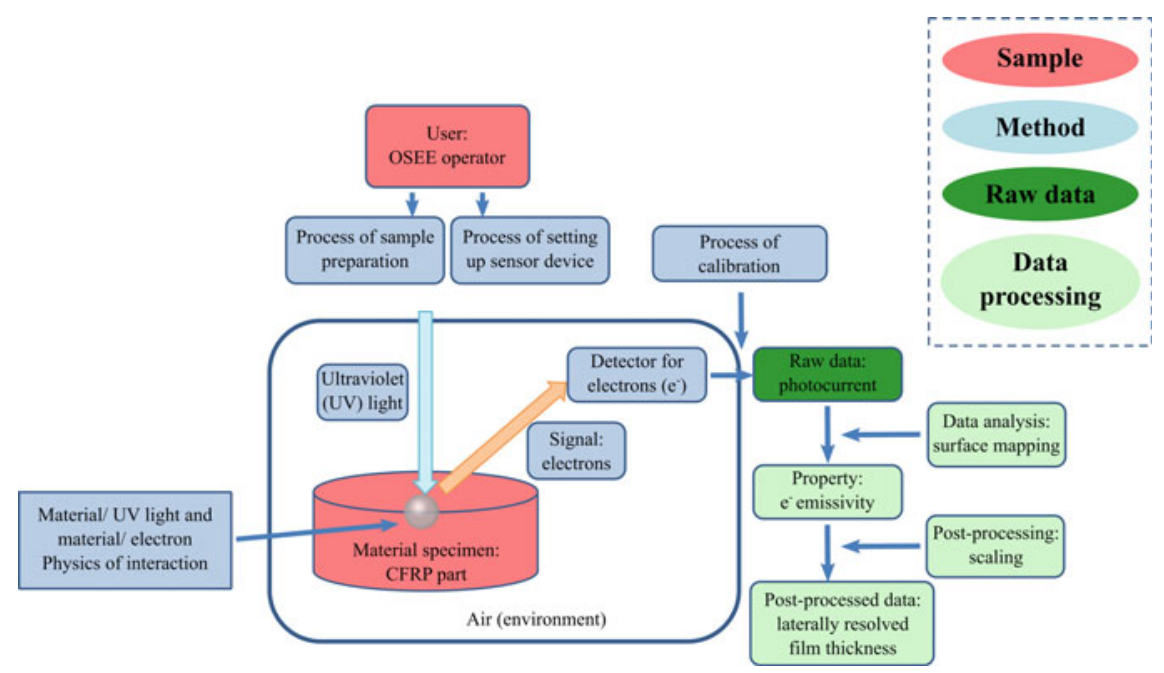

Fig. 6.4 Example of a CHADA (CHAracterization DAta) documentation implying metadata aspects, probe/surface interactions, and data processing steps for the inspection of a CFRP part using the optically stimulated electron emission (OSEE) ENDT technique; composed following the approach described by Romanos et al. [18]

complementary to rather data-driven regression methods that aim to find mathematical structures for the analysis of experimental information [24]. Combining both approaches systematically will be greatly facilitated by the interoperability of models and data.

We start by assessing further details of the recently presented systematic CHADA documentation shown in Fig. 6.4 for the required user responsibilities, comprising several aspects common to multiple-stage analytical processes. Both materialoriented, i.e. operand-related, and instrumental, i.e. operator-related, competencies are highlighted. In addition to setting up a specimen to be analyzed and a sensor before a surface inspection, the CHADA approach stresses the importance of calibration in accessing the characteristic material-related properties from the measured properties of analytical probes. The concept detailed in Chap. 1 and implemented in the ensuing chapters is based on preparatively adjusting finely graduated and technologically relevant surface states to purposefully vary operand features. Starting from such a set of customized operands, we effectively established both the particular sensor response and the response of a standardized technologically approved test for assessing design-relevant (mechanical) features. In this regard, we would like to refer to the discussion in Chap. 3 on the OSEE findings for CFRP coupon specimens. We inferred from our investigations that the OSEE response is affected by both deposited contaminants and the lateral inhomogeneities of the CFRP surface following the manual grinding process. Furthermore, we discussed that de-coupling these two aspects might broaden the application scope of this ENDT technique. We accentuate here that such a de-coupling might be achieved through sophisticated data 
analysis or the investigation of materials with a well-known composition and structure. In a certain manner, this reasoning places a focus on monitoring and describing the progress and effects of the manual grinding process used for CFRP surface pretreatment. One lesson learned during the ComBoNDT research project was that the CFRP surface curvature imposes not only challenges related to the accessibility of the CFRP surface for the ENDT tool, but also issues related to a higher lateral inhomogeneity of the abrasive surface pre-treatment. In a physics-based approach, visualizing the latter might be achieved by locally accessing the distribution of exposed carbon fibres and capping by the matrix material as a position-dependent feature of the CFRP surface. Following the more pragmatic concept described in this book, such local variations intrinsically rather contributed to variations in a signal background or to a signal width, and we considered it essential to characterize and document these implications for each user case and contamination scenario based on carefully manufactured reference specimens. This is one of the reasons why we included their consideration within the ten heuristic quality assessment principles outlined in Chap. 1.

From the material application perspective, on the one hand, the ENDT datasets obtained for adherend surfaces in a harmonized way can (from a technical point of view) be communicated and immediately assessed along the process chain in those production and repair user cases that profit from adhesive bonding. On the other hand, the consequences of such relevant material-related information on the behaviour of an adhesive joint are not intuitively evident. Therefore, it is essential to link the inspection data to the material features that are relevant to the design, and eventually the durability, of the device or structural part incorporating the inspected adherend. As outlined in Chap. 1, this link is provided by applying the concept developed in the ComBoNDT project. For example, enhancing the procedure described by Tornow et al. [25], Moutsompegka et al. demonstrated this conceptual approach by linking OSEE-based ENDT data for one scenario of one user case [26] with design-relevant fracture toughness findings. In Fig. 6.5, which is based on test results obtained in technologically relevant user cases during the ComBoNDT, we exemplarily demonstrate that findings from the fracture toughness tests qualitatively and even quantitatively go along with the scenario-specific ENDT inspection datasets obtained from surface monitoring of CFRP adherends with OSEE before the bonding process.

\subsection{Sensor Systems, Arrays and Networks for Assessing MoL Data}

Following a life cycle approach, a product's beginning-of-life (BoL) period comprises the manufacturing phase, which is succeeded by the middle-of-life (MoL) period. It is attractive to apply non-destructive evaluation (NDE) and ENDT techniques developed for adhesive bonding processes not only during manufacture, but 
(a)

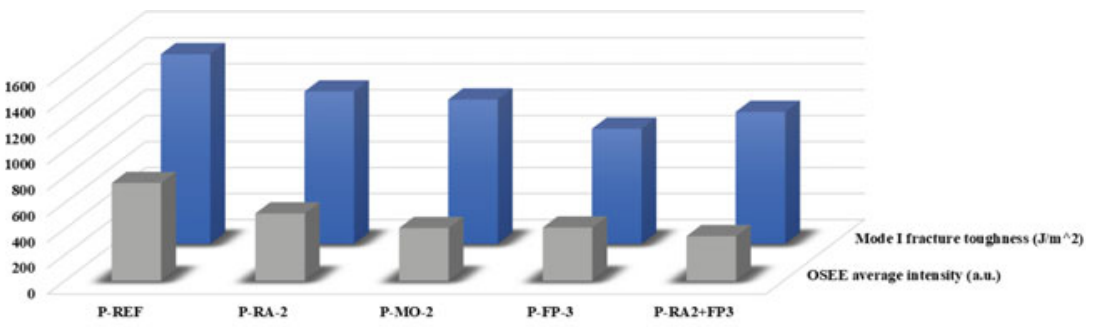

(b)

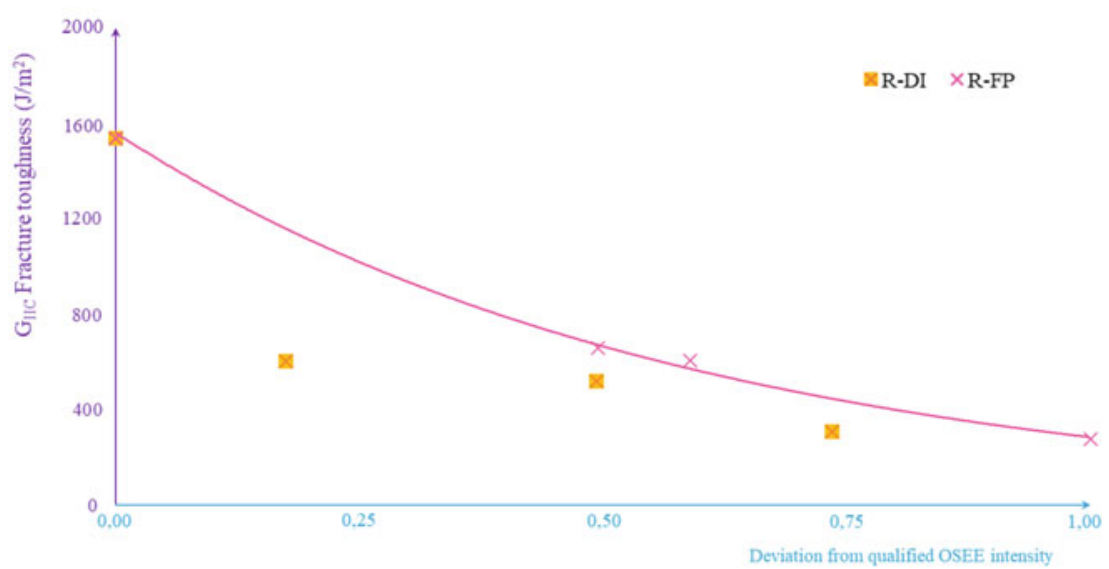

Fig. 6.5 Exemplary representation of outcomes from the ComBoNDT research project showing links between the fracture toughness $\left(\mathrm{G}_{I C}\right.$ or $\left.\mathrm{G}_{I I C}\right)$ measured for CFRP adhesive joints and normalized OSEE intensities measured for the surface of one of the adherends introduced in the bonding process. Distinct scenarios representing a deviation from a qualified surface pre-treatment process were considered for a production $(\mathrm{P})$ and a repair $(\mathrm{R})$ user case. a Reduced $\mathrm{G}_{I C}$ values qualitatively go along with reduced OSEE intensities; $\mathbf{b}$ characteristic curves quantitatively depict scenariospecific connections between the design-relevant mechanical joint feature $\mathrm{G}_{I I C}$ and the ENDT signal measured using OSEE

also during repair, which is a conceivable user case during a product's MoL. Procedures for monitoring the surface quality of adherends are tailored to open adhesive joints, i.e. the process steps before the application of the adhesive. Beyond that, the approaches sketched in Fig. 1.4 (in Chap. 1) with respect to manufacturing processes encompass bonding quality assessment, which is immediately relevant for all life cycle phases that are governed by a closed adhesive joint, for instance, in the case of composite parts. Accordingly, cyber-physical systems (CPS) relevant for ENDT during the BoL period are also technically relevant for the MoL of a product, facilitating the networking between the machines and equipment as part of the physical world and the cyberspace or internet as part of the digitalized virtual world. As outlined in the previous chapters, sensing systems based on sensors facilitate 
the observation and documentation of processes or the characteristics of a material object that are not visible to the human eye and cannot be recorded with other human senses [27]. As highlighted by Grosse and discussed in the previous chapters, a sensor acts as a filter that can significantly influence the way humans look at processes and material properties, e.g. by introducing more or fewer distortions and alterations to the data, and thus, the transmission of information. These considerations are featured in Fig. 1.4 (Chap. 1) by highlighting how relevant the quality of cyber-physical interaction is for the quality (assessment) of the output provided by a bonding process.

Moreover, the application of ENDT systems is conceptually closely related to the user cases expected during the MoL period of adhesive joints, such as those comprising the service aspects of MRO. In this context, conventional NDT procedures, such as ultrasonic or X-ray-based inspection or thermography, are rather mature technologies, yet they are intermittently applied. Their reliability has been extensively demonstrated, for instance, in testing aerospace components to detect defects, cracks, corrosion or other degradation features as part of quality assurance and in-service inspection [28]. Following this statement, and also in accordance with the previous chapters, recent directions comprise automated, fast and cost-effective in-service and in-situ monitoring, e.g. of aircraft. However, such conventional NDT procedures rely on access to structural parts with external probes or equipment, thus they are not suitable for condition-based maintenance concepts, and they require human intervention [29]. Beyond that and over an extended period of time, permanently installed SHM systems facilitate the application of non-destructive evaluation and testing methods to survey the state and condition of load-bearing structural components, primarily motivated by further enhancing safety [30, 31]. Following SAE Standard ARP6461, SHM is defined as "the process of acquiring and analyzing data from on-board sensors to evaluate the health of a structure" [29]. To achieve this, SHM systems include three key elements [29] and three steps [32]. Following Güemes et al., these elements comprise first, a network of sensors that are permanently attached to the structure, which is essential for performing automated inspections; second, on-board data handling and computing facilities for processing data in real-time; and third, algorithms that compare recently acquired data with stored data on the pristine structure. In this way, after correcting for environmental factors, a damage index may be calculated and information about damage existence, localization and type may be provided. Furthermore, damage severity may be quantified, and the residual life may be predicted [32]. Such prognosis is closely linked to diagnosis, or the evaluation of structural health, which is the main objective of SHM; prognosis and diagnosis conceptually differ in the tools they use: prognosis is mainly focused on statistical analysis, while diagnosis is more related to sensors, signal processing and algorithms for damage identification [29]. Güemes et al. record that some authors consider prognosis to be the fifth level of SHM, with levels one to four being related to damage detection, localization, classification and quantification of damage, respectively. Accordingly, Haldar [33] attributes the latter two development levels, with respect to the technical sophistication of SHM systems, to the 
assessment of damage and the prediction of development. Augustin et al. underline that the development and integration of a suitable sensor network is a major challenge facing scientists and engineers of SHM systems [34]. They list eligible methods for SHM systems, including fibre optic sensors, piezoceramic transducers for Lamb wave excitation and collection, vibration-based methods, acoustic emission techniques and electrical conductivity methods. In the following, we highlight recent achievements in diagnosis and provide insights for guided wave propagation, electromechanical impedance or fibre Bragg grating sensors.

An aspect that is inherent to the development and advancement of sensor systems and networks for NDT is considering the comprehensive applicability of such procedures in the distinct processes and environments of various technological sectors. For instance, approaches based on guided wave propagation, as well as electromechanical impedance, in composite materials and material joints have been widely investigated for the purpose of damage assessment. Here, we consider damage as not only an extensive loss of material functionality, but also an implied degradation of relevant material features as compared to the starting state. Widening this scope even further towards assessing this starting state, as well as the material surface, we proposed, tested and established both of these techniques for the non-destructive evaluation of adherends and adhesively bonded CFRP parts following the production and repair processes investigated within the frame of the ENCOMB and ComBoNDT joint research projects. Chapter 3 presented the ENDT results of the guided wavebased studies for surface characterization, and Chap. 4 described the electromechanical impedance results for adhesive bond evaluation. Taking this into consideration, the approaches of damage detection and the assessment of the adhesive bond state may be combined using the same sensor systems and networks. However, in this context, we must still answer the question of how to deploy the sensors. As one answer, designing a correct—and admittedly complex — network is the first step to success. Numerous sensor network solutions are being developed in the academic environment, and over the last decade, some of the results have been further developed and commercialized by companies. One of the better-known examples is the Stanford Multiactuator-Receiver Transduction (SMART) layer concept, which was conceived at Stanford University and later commercialized by Acellent Technologies, Inc. [35]. The SMART layer concept allows for the deployment of a sensor network on a surface, as well as embedding it in a layered composite as one of the plies in the layerwise composite structure. Quite recently, a new approach to sensor networks was proposed: the stretchable sensor network [36]. The leading idea behind this is to have a network that can be deployed across a large area yet remains compact prior to its deployment. Although such ideas do not provide an answer to the problem of how the sensors should be deployed on the structure, the placement of the sensors can be tackled by various approaches. Some of the popular strategies are based on evolutionary algorithms, such as genetic algorithms [37], whereby elliptic-based damage localisation algorithms may be used as a basis for optimisation. Here, the locations of actuating and recording transducers correspond to the ellipse foci, and the circumference of the ellipse informs about the source of wave reflection. If there is no damage or other discontinuity, then no reflection occurs. The 
type of transducer network determines the positioning of these ellipses. A different approach to the concept of a sensor network involves placing two transducers at each network node - an actuating and a receiving transducer [38]. Such transducer placement causes the resulting ellipses to be almost circular. Distributed systems are used not only for damage location, but also in damage identification for crack shape reconstruction [39]. A very informative review of the problem of optimal sensor placement was presented by Ostachowicz et al. [40]. Meanwhile, the sensor placement oriented towards hot spot monitoring is based on a tomography approach in which a localized area surrounded by sensors is monitored [41]. This was successfully applied for the debonding of stiffening elements from the skin of a composite wing [42], and here we can find a good link between damage detection and the assessment of adhesive bonds. In contrast to distributed networks and tomography approaches, there have also been attempts to employ a concentrated network of transducers. Researchers have analyzed various sensor placements in order to identify the optimum solutions. One of the most popular solutions has a circular shape, and there are examples with 13 [43] or 16 [44] sensors forming the circle. A special case of concentrated arrays is represented by the phased arrays that use a special method of signal processing to simulate wave interference. This effect allows for amplifying waves reflecting from an area of damage [30]. The simple phased array is linear in shape and has been successfully used for locating damage in aluminium specimens both with and without surface curvature [45], as well as in composites [46]. In summary, based on the achievements presented within the scope of this book and in published studies on sensor networks [35-40], we suggest that the same sensor networks may be used for both damage and adhesive bond assessment. Only the signal processing approaches are different for both applications and need to be tailored to the specific task. Such customisation will thus require knowledge of the respective technological environment, as well as standards for communication within the greater supervisory control and data acquisition (SCADA) network. SCADA has become the standard and is currently in operation on wind farms to gather measurements of wind speed, bearing and oil temperatures, voltage, power produced and other variables [47]. Therefore, the integration of new damage and adhesive bonding monitoring techniques should be integrated into the SCADA scheme.

In SHM applications ranging from aircraft to civil infrastructure, fibre optic sensors (FOS) are potentially very well suited to measuring variables such as temperature, electrical current, strain and pressure, even when working in electromagnetic fields, at high temperature or humidity, or in an aggressive chemical environment; also, they have demonstrated surprisingly high reliability [48]. During their life cycle, many adhesively bonded structures experience long-term dynamic loads in the MoL phase, which might lead to the preliminary performance reduction of bonded joints exposed to relatively low load levels [34]. Recently, we reported on the intrusive effect of adhesive bond strength and adhesive layer thickness and quality when embedding optical glass fibres carrying fibre Bragg grating sensors directly in the $0.1 \mathrm{~mm}$ thin adhesive bond formed by the structural and repair film adhesives applied in CFRP joints [49]. We showed that influences on the fatigue limit of the tested film adhesive joints were negligible whenever optical glass fibres with total diameters smaller than 
$100 \mu \mathrm{m}$ were embedded. Having information on structural events or states available at arbitrary times may reduce the occurrence of unscheduled events, facilitate preventive maintenance [50], and pave the way towards condition-based structural maintenance [51].

Summing up, the research on and development of reliable SHM systems is of great importance. Conventional NDT provides non-destructive and effective tools for detecting and localizing damage in a predefined area and can form part of an SHM system; however, SHM is much more than NDT [52]. For SHM purposes, fibrebased sensors and smart materials may be integrated permanently into the monitored structures and the data may be collected continuously or at least periodically [53]. The collected data are then transferred to a computer system, where they are processed and analyzed for possible defects or changes in the material characteristics, e.g. damage accumulation, in almost real-time [52]. This is crucial not solely for FOSbased sensing because the collected data have little value without proper analysis and the extraction of information about the health state of the structure [33]. In fact, one of the major challenges of SHM systems is related to damage identification and assessment taking place in a mostly unsupervised learning mode [54]. Moreover, the scope of an SHM system enfolds different monitoring and learning levels. On the lowest level, the SHM system only monitors a certain structure or the assembly of a system (e.g. the wing of an aircraft), while on an advanced level the whole structural system is involved (e.g. the whole aircraft). On an even higher level, the data from all individual structural systems (e.g. all aircraft) are pooled and analyzed for recurring damage patterns or principles that can be applied to the whole fleet [52], as outlined in Fig. 6.6.

The successful integration of an SHM system leads to prolonged maintenance intervals, a reduction in maintenance costs, a resource-conserving exchange of wear parts, and a further increase in safety [34].

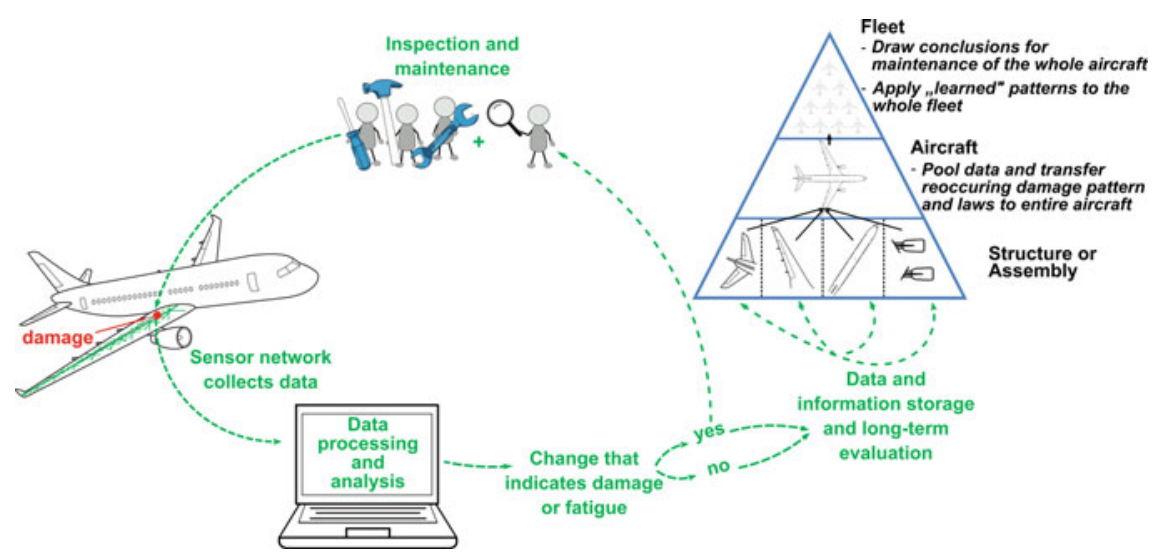

Fig. 6.6 Different monitoring and learning levels in the scope of an SHM system facilitated by data integration 


\subsection{Synopsis}

Encouraged by the foreseeable availability of high-quality data from the advanced ENDT procedures presented in the previous chapters of this book and inspired by the numerous impulses from fruitfully cooperating in a joint research project, we have indicated in this last chapter that we might already project some facets of possible answers onto central future questions, such as:

- What are the drivers in worldwide digitalization and data exchange along the life cycle of a (material) product?

For instance, sustainable and holistic life cycle product management will benefit from high-quality and relevant information provided to managers because there is some truth in the saying "You can't manage what you can't measure" [55].

- How can we contribute ENDT datasets from quality assessment as an input to worldwide workflows among the involved stakeholders?

On the one hand, after a bonding process during BoL (e.g. manufacturing), material-related ENDT metadata and data may be available during MoL preventive maintenance and thereby allow for an evaluation of changes in the material state. On the other hand, in the case of MoL repair, a tailored device facilitating local ENDT may guide decisions by remote experts for cases of required cleaning on demand.

- How can quality assurance of local material-related transformation processes be promoted by receiving from external sources an input of material-related data gathered during earlier or subsequent life cycle phases?

For example, in a bonding process during BoL or MoL (e.g. repair), the quality assessment may be guided by data obtained during the manufacturing of the adherends or during the application of the bonded joint (e.g. using SHM).

All of these questions may be posed for various types of material operands that are changed (e.g., joined) by distinct operators during a broad range of, e.g. adhesionbased, processes within the life cycle of products in different applications. This means that there is still a wealth of aspects and phenomena to be explored. Notwithstanding this, we are convinced that in facing these challenges, the advancement of ENDT tools and procedures achieved in the ComBoNDT research project, as well as FAIR data structures, lay a good technological and knowledge basis that is open for application!

\section{References}

1. McMillan AJ, Swindells N, Archer E et al (2017) A review of composite product data interoperability and product life-cycle management challenges in the composites industry. Adv Manuf: Polym Compos Sci 3:130-147. https://doi.org/10.1080/20550340.2017.1389047

2. OntoTRANS "Ontology driven Open Translation Environment" (2020-2024) Project funded from the European Union's Horizon 2020 research and innovation programme under grant agreement No 862136. https://ontotrans.eu/ 
3. ComBoNDT "Quality assurance concepts for adhesive bonding of aircraft composite structures by advanced NDT" (2015-2018) Project funded from the European Union's Horizon 2020 research and innovation programme under grant agreement No 636494. https://cordis.europa. eu/project/id/636494

4. Klöpffer W, Renner I (2008) Life-cycle based sustainability assessment of products. In: Schaltegger S, Bennett M, Burritt RL et al (eds) Environmental management accounting for cleaner production, vol 24, 1st edn. Springer, Netherlands, pp 91-102. https://doi.org/10.1007/978-14020-8913-8_5

5. Sonnemann G, Vigon B (eds) (2011) Global guidance principles for life cycle assessment databases - a basis for greener processes and products. United Nations Environment Programme

6. Calado EA, Leite M, Silva A (2019) Integrating life cycle assessment (LCA) and life cycle costing (LCC) in the early phases of aircraft structural design: an elevator case study. Int J Life Cycle Assess 24:2091-2110. https://doi.org/10.1007/s11367-019-01632-8

7. Otte JN, Kiritsi D, Ali MM et al (2019) An ontological approach to representing the product life cycle. AO 14:179-197. https://doi.org/10.3233/AO-190210

8. Hermann M, Pentek T, Otto B (2016) Design principles for industrie 4.0 scenarios. In: 201649th Hawaii international conference on system sciences (HICSS). IEEE, pp 3928-3937. https:// doi.org/10.1109/HICSS.2016.488

9. Ghedini E, Friis J, Hashibon A et al (2020) EMMO an ontology for applied sciences. http:// emmc.info/emmo-info/

10. Ghedini E, Friis J, Hashibon A et al (2020) European materials \& modelling ontology (EMMO). http://github.com/emmo-repo/EMMO/

11. VIMMP "Virtual Materials Market Place" (2018-2021) Project funded from the European Union's Horizon 2020 research and innovation programme under grant agreement No 760907. https://www.vimmp.eu/

12. Horsch MT, Chiacchiera S, Seaton MA et al (2020) Ontologies for the virtual materials marketplace. Künstl Intell 34:423-428. https://doi.org/10.1007/s13218-020-00648-9

13. MarketPlace "Materials Modelling Marketplace for Increased Industrial Innovation" (20182022) Project funded from the European Union's Horizon 2020 research and innovation programme under grant agreement No 760173. https://www.the-marketplace-project.eu/

14. Cavalcanti WL, Noeske P-LM (2014) Investigating dynamic interactions by multi-scale modelling: from theory to applications. In: Springborg M, Joswig J-O (eds) Chemical modelling, vol 11. Royal Society of Chemistry, Cambridge, pp 175-200. https://doi.org/10. 1039/9781782620112-00175

15. Rottenkohlber M (2010) Industrielle 3D Sensorik - Wo geht die Reise hin? INSPECT 7:12-14

16. Tornow C, Brune K (2019) Vorhabenbezeichnung: HANOB - "Entwicklung Referenzsystem zur handgeführten Oberflächen-Benetzungsprüfung": Abschlussbericht gem. NKBF 98: Berichtszeitraum: 01.05.2016 bis 31.10.2018. Fraunhofer-Institut für Fertigungstechnik und Angewandte Materialforschung (IFAM)

17. Cherrier C, Hanning T (2019) Vorhabenbezeichnung: HANOB - "Entwicklung Referenzsystem zur handgeführten Oberflächen-Benetzungsprüfung" : Abschlussbericht gem. NKBF 98 : Laufzeit des Vorhabens: 01.05.2016 bis 30.04.2018 verlängert zum 30.10.2018. Automation $\mathrm{W}+\mathrm{R} \mathrm{GmbH}$

18. Romanos N, Kalogerini M, Koumoulos EP et al (2019) Innovative data management in advanced characterization: implications for materials design. Mater Today Commun 20:100541. https://doi.org/10.1016/j.mtcomm.2019.100541

19. de Baas $\mathrm{AF}$ (2017) What makes a material function? Let me compute the ways. Note: This document is commonly referred to as the Review of Materials Modelling (RoMM). EU Publications Office, Luxembourg. https://doi.org/10.2777/21919

20. Simperler A, Goldbeck G, Sebastiani M (2019) MODA and CHADA: terminology and standardized documentation for materials modelling and characterization; EuroNanoForum 2019 (Nanotechnology and Advanced Materials Progress Under Horizon2020 and Beyond), Bucharest, Romania, 12-14 June 
21. EMMC ASBL, The European Materials Modelling Council (2019). https://emmc.eu/

22. EMMC-CSA "European Materials Modelling Council" (2016-2019) Project funded from the European Union's Horizon 2020 research and innovation programme under grant agreement No 723867. https://emmc.info/

23. EMCC, European Materials Characterisation Council (2020). http://www.characterisation.eu/

24. Gomes FA, de O Assis A, R. da C Reis M et al (2017) Proposal of heuristic regression method applied in descriptive data analysis: case studies. TEEE 2:51. https://doi.org/10.22149/teee.v2i 2.105

25. Tornow C, Schlag M, Lima LCM et al (2015) Quality assurance concepts for adhesive bonding of composite aircraft structures-characterisation of adherent surfaces by extended NDT. J Adhes Sci Technol 29:2281-2294. https://doi.org/10.1080/01694243.2015.1055062

26. Moutsompegka E, Tserpes K, Noeske M et al (2019) Experimental investigation of the effect of pre-bond contamination with fingerprints and ageing on the fracture toughness of composite bonded joints. Appl Compos Mater 26:1001-1019. https://doi.org/10.1007/s10443-019-097 63-9

27. Grosse CU (2013) Evolution of NDT methods for structures and materials: some successes and failures. In: Büyüköztürk O, Taşdemir MA, Güneş $\mathrm{O}$ et al (eds) Non-destructive testing of materials and structures. Springer, Netherlands, Dordrecht, pp 3-18. https://doi.org/10.1007/ 978-94-007-0723-8_1

28. Purna Chandra Rao B (2017) Non-destructive testing and damage detection. In: Prasad NE, Wanhill RJH (eds) Aerospace materials and material technologies. Springer Singapore, Singapore, pp 209-228. https://doi.org/10.1007/978-981-10-2143-5_11

29. Güemes A, Fernandez-Lopez A, Pozo AR et al (2020) Structural health monitoring for advanced composite structures: a review. J Compos Sci 4:13. https://doi.org/10.3390/jcs4010013

30. Giurgiutiu V (2014) Structural health monitoring with piezoelectric wafer active sensors, 2nd edn. AP Academic Press/Elsevier, Amsterdam. https://doi.org/10.1016/C2013-0-00155-7

31. Lehmhus D, Busse M (2018) Structural health monitoring (SHM). In: Bosse S, Lehmhus $\mathrm{D}$, Lang $\mathrm{W}$ et al (eds) Material-integrated intelligent systems - technology and applications. Wiley-VCH Verlag GmbH \& Co, KGaA, Weinheim, Germany, pp 529-570. https://doi.org/ 10.1002/9783527679249.ch22

32. Askaripour K, Zak A (2019) A survey of scrutinizing delaminated composites via various categories of sensing apparatus. J Compos Sci 3:95. https://doi.org/10.3390/jcs3040095

33. Haldar A (ed) (2013) Health assessment of engineered structures: bridges, buildings and other infrastructures. World Scientific, New Jersey, London, Singapore, Beijing, Shanghai, Hong Kong, Taipei, Chennai. https://doi.org/10.1142/8628

34. Augustin T, Karsten J, Kötter B et al (2018) Health monitoring of scarfed CFRP joints under cyclic loading via electrical resistance measurements using carbon nanotube modified adhesive films. Compos A Appl Sci Manuf 105:150-155. https://doi.org/10.1016/j.compositesa.2017. 11.015

35. Qing XP, Beard SJ, Kumar A et al (2007) Built-in sensor network for structural health monitoring of composite structure. J Intell Mater Syst Struct 18:39-49. https://doi.org/10.1177/104 5389X06064353

36. Guo Z, Kim K, Salowitz N et al (2018) Functionalization of stretchable networks with sensors and switches for composite materials. Struct Health Monit 17:598-623. https://doi.org/10. $1177 / 1475921717709632$

37. Soman R, Malinowski P (2019) A real-valued genetic algorithm for optimization of sensor placement for guided wave-based structural health monitoring. J Sens 2019:1-10. https://doi. org/10.1155/2019/9614630

38. Qiang W, Shenfang Y (2009) Baseline-free imaging method based on new PZT sensor arrangements. J Intell Mater Syst Struct 20:1663-1673. https://doi.org/10.1177/1045389X0 9105232

39. Quek ST, Tua PS, Jin J (2007) Comparison of plain piezoceramics and inter-digital transducer for crack detection in plates. J Intell Mater Syst Struct 18:949-961. https://doi.org/10.1177/ 1045389X06071435 
40. Ostachowicz W, Soman R, Malinowski P (2019) Optimization of sensor placement for structural health monitoring: a review. Struct Health Monit 18:963-988. https://doi.org/10.1177/147592 1719825601

41. Wang D, Ye L, Lu Y et al (2010) A damage diagnostic imaging algorithm based on the quantitative comparison of Lamb wave signals. Smart Mater Struct 19:65008. https://doi.org/ 10.1088/0964-1726/19/6/065008

42. Fasel TR, Todd MD (2010) An adhesive bond state classification method for a composite skinto-spar joint using chaotic insonification. J Sound Vib 329:3218-3232. https://doi.org/10.1016/ j.jsv.2010.02.009

43. Kudela P, Ostachowicz W, Żak A (2008) Damage detection in composite plates with embedded PZT transducers. Mech Syst Signal Process 22:1327-1335. https://doi.org/10.1016/j.ymssp. 2007.07.008

44. Stepinski T, Engholm M (2009) Piezoelectric circular array for structural health monitoring using plate waves. In: Proceedings of the 7th international workshop on structural health monitoring, pp 1050-1056

45. Yu L, Santoni-Bottai G, Xu B et al (2008) Piezoelectric wafer active sensors for in situ ultrasonic-guided wave SHM. Fatigue Fract Eng Mater Struct 31:611-628. https://doi.org/doi:10. 1111/j.1460-2695.2008.01256.x

46. Sundararaman A, Adams DE, Rigas EJ (2005) Biologically inspired structural diagnostics through beamforming with phased transducers arrays. Int J Eng Sci 43:756-778. https://doi. org/10.1016/j.ijengsci.2004.12.008

47. Antoniadou I, Dervilis N, Papatheou E et al (2015) Aspects of structural health and condition monitoring of offshore wind turbines. Philos Trans R Soc A: Math Phys Eng Sci 373. https:// doi.org/10.1098/rsta.2014.0075

48. Meier U, Brönnimann R, Anderegg P et al (2013) 20 years of experience with structural health monitoring of objects with CFRP components. In: Büyüköztürk $\mathrm{O}$, Taşdemir MA, Güneş $\mathrm{O}$ et al (eds) Non-destructive testing of materials and structures. Springer, Netherlands, Dordrecht, pp 959-976. https://doi.org/10.1007/978-94-007-0723-8_136

49. Grundmann N, Brüning $H$, Tserpes $K$ et al (2020) Influence of embedding fiber optical sensors in CFRP film adhesive joints on bond strength. Sensors (Basel) 20. https://doi.org/10.3390/s20 061665

50. Speckmann H (2008) Structural health monitoring (SHM). In: International maintenance review board policy board (IMRBPB). EASA

51. Lewis A (2008) Structural health management/monitoring. In: International maintenance review board policy board (IMRBPB). EASA

52. Balageas D, Fritzen C-P, Güemes A (2006) Structural health monitoring. Wiley Online Library. https://doi.org/10.1002/9780470612071

53. Trilaksono A, Watanabe N, Hoshi $\mathrm{H}$ et al (2013) Continuous damage monitoring of a thin composite structural with mismatched stiffener in a combined joint using fiber Bragg grating under tension and three-point loading. OJCM 03:63-87. https://doi.org/10.4236/ojcm.2013. 33008

54. Farrar CR, Worden K (2010) An introduction to structural health monitoring. In: Deraemaeker A (ed) New trends in vibration based structural health monitoring. Springer, Wien. https://doi. org/10.1098/rsta.2006.1928

55. Baumast A (2017) Geschichte und aktuelle Herausforderungen des betrieblichen Nachhaltigkeitsmanagements. In: Pape J, Baumast A, Weihofen S et al (eds) Betriebliche Nachhaltigkeitsleistung messen und steuern: Grundlagen und Praxisbeispiele. Verlag Eugen Ulmer, Stuttgart, pp 18-30 
Open Access This chapter is licensed under the terms of the Creative Commons Attribution 4.0 International License (http://creativecommons.org/licenses/by/4.0/), which permits use, sharing, adaptation, distribution and reproduction in any medium or format, as long as you give appropriate credit to the original author(s) and the source, provide a link to the Creative Commons license and indicate if changes were made.

The images or other third party material in this chapter are included in the chapter's Creative Commons license, unless indicated otherwise in a credit line to the material. If material is not included in the chapter's Creative Commons license and your intended use is not permitted by statutory regulation or exceeds the permitted use, you will need to obtain permission directly from the copyright holder.

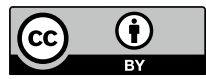

